\title{
Adaptive radiation and burst speciation of hillstream cyprinid fish Garra in African river
}

\author{
Boris Levin ${ }^{1}$, Evgeniy Simonov ${ }^{2}$, Paolo Franchini ${ }^{3}$, Nikolai Mugue ${ }^{4}$, Alexander Golubtsov ${ }^{5}$, \\ and Axel Meyer ${ }^{3}$
}

${ }^{1}$ I D Papanin Institute of Biology of Inland Waters RAS

${ }^{2}$ Institute of Environmental and Agricultural Biology (X-BIO), University of Tyumen, Tyumen

${ }^{3}$ University of Konstanz

${ }^{4}$ Kol'tsov Institute of Developmental Biology, Russian Academy of Sciences

${ }^{5} \mathrm{~A}$ N Severtsov Institute of Ecology and Evolution RAS

May 12, 2021

\begin{abstract}
Adaptive radiation of fishes was long thought to be possible only in lacustrine environments. Recently, several studies have shown that also riverine and stream environments provide the ecological opportunity for adaptive radiation. In this study, we report on a riverine adaptive radiation of six ecomorphs of cyprinid hillstream fishes of the genus Garra in a river located in the Ethiopian Highlands in East Africa. Garra are predominantly highly specialized algae-scrapers with a wide distribution ranging from Southeastern Asia to Western Africa. However, adaptive phenotypic diversification in mouth type, sucking disc morphology, gut length and body shape have been found among these new species in a single Ethiopian river. Moreover, we found two novel phenotypes of Garra ('thick-lipped' and 'predatory') that were not described before in this species-rich genus (>160 species). Mitochondrial and genome-wide data suggest monophyletic, intra-basin evolution of Garra phenotypic diversity with signatures of gene flow from other local populations. Although sympatric ecomorphs are genetically distinct and can be considered to being young species as suggested by genome-wide SNP data, mtDNA was unable to identify any genetic structure suggesting a recent and rapid speciation event. Furthermore, we found evidence for a hybrid origin of the novel 'thick-lipped' phenotype, as being the result of the hybridization of two other sympatrically occurring species. Here we highlight how, driven by ecological opportunity, an ancestral trophically highly specialized lineage is likely to have rapidly adaptively radiated in a riverine environment, and that this radiation was promoted by the evolution of novel feeding strategies.
\end{abstract}

\section{Hosted file}

AMed_Garra_Sore_final_Figures_down.pdf available at https://authorea.com/users/413433/ articles/521748-adaptive-radiation-and-burst-speciation-of-hillstream-cyprinid-fishgarra-in-african-river 